The relationship between local scalp skin temperature and cutaneous perfusion during scalp cooling

This article has been downloaded from IOPscience. Please scroll down to see the full text article. 2007 Physiol. Meas. 28829

(http://iopscience.iop.org/0967-3334/28/8/006)

View the table of contents for this issue, or go to the journal homepage for more

Download details:

IP Address: 130.89.112.86

The article was downloaded on 28/03/2011 at 10:52

Please note that terms and conditions apply. 


\title{
The relationship between local scalp skin temperature and cutaneous perfusion during scalp cooling
}

\author{
Francis-Paul E M Janssen ${ }^{1,3}$, Vinayakrishnan Rajan ${ }^{2,3}$, \\ Wiendelt Steenbergen ${ }^{2}$, Gerard M J van Leeuwen ${ }^{1}$ \\ and Anton A van Steenhoven ${ }^{1}$ \\ ${ }^{1}$ Department of Biomedical Engineering, Eindhoven University of Technology, PO Box 513, \\ 5600 MB Eindhoven, The Netherlands \\ 2 Faculty of Science and Technology, Biophysical Engineering Group, University of Twente, \\ PO Box 217, NL-7500AE Enschede, The Netherlands \\ E-mail: f.e.m.janssen@tue.nl
}

Received 23 February 2007, accepted for publication 30 May 2007

Published 6 July 2007

Online at stacks.iop.org/PM/28/829

\begin{abstract}
Cooling the scalp during administration of chemotherapy can prevent hair loss. It reduces both skin blood flow and hair follicle temperature, thus affecting drug supply and drug effect in the hair follicle. The extent to which these mechanisms contribute to the hair preservative effect of scalp cooling remains unknown. The purpose of this study was to establish a relationship between local scalp skin temperature and cutaneous blood flow during scalp cooling. We measured skin temperature and cutaneous perfusion during a cooling and re-warming experiment. Experiments on a single subject showed that the measurements were reproducible and that the response was identical for the two positions that were measured. Inter-subject variability was investigated on nine subjects. We found that for the first $10{ }^{\circ} \mathrm{C}$ of cooling, perfusion of the scalp skin decreases to below $40 \%$. Perfusion can be further reduced to below $30 \%$ by a few degrees more cooling, but a plateau is reached after that. We found that a generally accepted relation in thermal physiology between temperature and perfusion (i.e. $Q_{10}$ relation) does not describe the data well, but we found an alternative relation that describes the average behavior significantly better.
\end{abstract}

Keywords: scalp cooling, laser Doppler perfusion monitoring, skin temperature 


\section{Introduction}

Chemotherapy as a cancer treatment often leads to partial or complete hair loss, one of the most feared side effects of cancer therapy (Cash 2001). Cooling the scalp during chemotherapy treatment can reduce or even prevent this hair loss (Protière et al 2002). Two possible mechanisms explain how scalp cooling might contribute to hair preservation (Grevelman and Breed 2005). First, a reduced blood flow to the cutaneous tissue during cooling could lead to a reduced drug supply to the hair follicle. Second, a reduction in cutaneous cell metabolism as a response to the hypothermia could simply make hair follicles less susceptible to drug damage. Currently, the dependence of both these mechanisms on temperature is unknown and hence the cooling protocol may not be optimal. Also there are insufficient data available on the relationship between the local skin temperature and local cutaneous blood perfusion, and existing data for the scalp skin during scalp cooling (Bülow et al 1985) are brief and lack accuracy. Bülow et al (1985) used a xenon washout technique on ten subjects to identify pre-cooling, cooling and post-cooling perfusion. During cooling, perfusion reduced to about $20 \%$ of the pre-cooling level. Unfortunately, standard deviations for pre-cooling and cooling perfusion were very high, leading to a high uncertainty in this minimum perfusion value. Also, no information is given about the relationship between minimum perfusion and scalp skin temperature. Such information is essential for quantitative modeling of human heat transfer and for a better understanding of human thermoregulatory processes.

The initial response of skin blood flow to skin cooling is cutaneous vasoconstriction. According to Thompson et al (2005), two different mechanisms are responsible for this vasoconstriction. The first mechanism is a response to whole-body skin cooling and is called reflex-mediated response. The second mechanism is a response to local skin cooling and is called locally mediated response. These two mechanisms of skin blood flow control are not mutually exclusive; often these mechanisms operate together during exposure to cold to maximize vasoconstriction. The exact mechanisms of the vasoconstrictor system are not yet well defined, but it is certain that the temperature of the blood vessels themselves plays an important role (Pèrgola et al 1993).

During scalp cooling, only the outermost part of the head experiences changes in temperature (Janssen et al 2005). The size of the affected region is approximately $2 \mathrm{~cm}$, and is too small to have any significant effect on core temperature. Therefore, we investigated whether a local relationship between temperature and perfusion exists during scalp cooling.

The cold provocation-induced changes in blood perfusion have been a subject of research for the diagnosis of Raynaud's phenomenon (Lau et al 1995, Popivanov et al 1999, Kanetaka et al 2004). These studies were mainly focused on the microcirculatory perfusion at different heat and cold provocations without looking at a periodic change of perfusion with temperature. Maniewski et al (1999) and Friedman et al (2007) followed perfusion by gradually decreasing temperature. They analyzed the perfusion level changes with temperature between healthy subjects and patients with Reynaud's phenomenon but did not attempt to relate the perfusion to temperature. Since these studies were performed on the basis of protocols which were relevant for the diagnosis for Reynaud's phenomenon and the measurements were performed by either cooling of the whole body or a hand the results cannot be interpreted for scalp cooling protocol for chemotherapy treatment.

The purpose of this study was to find the dependence of cutaneous blood flow on local scalp skin temperature during scalp cooling, in a setting that is relevant for clinical practice. For this, the scalp was cooled using a commercially available scalp cooling device, and temperature and perfusion were measured using thermocouples and laser Doppler perfusion probes, respectively. It was hypothesized that (i) a relationship exists between local skin 


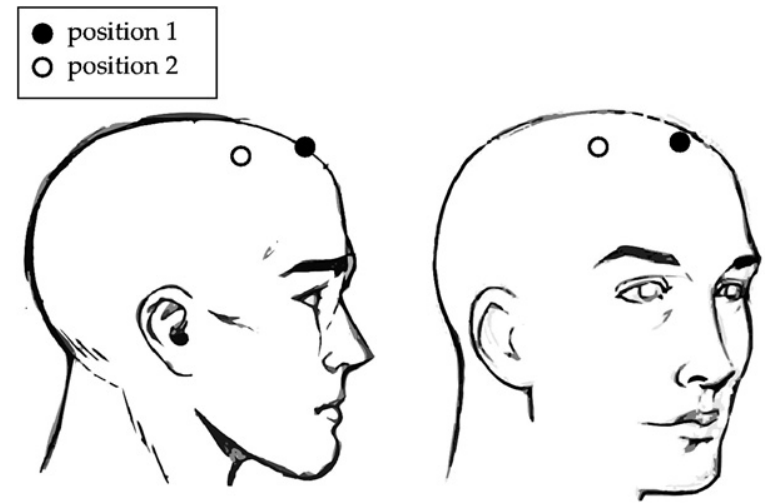

Figure 1. A cartoon of the positions of the probe on scalp skin, as used in measurements. Position 1 represents the mid-front of the hairline and position 2 a top-right spot on the hairline.

temperature and cutaneous blood flow during scalp cooling and (ii) that this relationship is independent of the subject regarded.

\section{Materials and methods}

\subsection{Subjects}

Experiments were performed on nine subjects ( 7 male, 2 female; age 24-43) after informed consent. Subjects were not screened for any cardiovascular or neurological disorder. For each subject a single experiment was performed using the mid-front of the hairline (referred to as position 1). On one subject (male, age 26) inter-position and inter-experiment variability were investigated using both the mid-front of the hairline and a top-right position in the hairline (referred to as position 2). Figure 1 illustrates the location of positions 1 and 2 on the head. For each probe position, three experiments were performed with an interval between experiments of $24 \mathrm{~h}$.

\subsection{Blood flow measurements}

Cutaneous blood flow was measured with the laser Doppler flowmetry (LDF) technique. This is a noninvasive technique to monitor the blood microcirculatory flux, with a typical measurement depth of $1 \mathrm{~mm}$ for cutaneous measurements. The actual measurement depth varies with tissue optical properties, and the averaged depth for typical tissue optical properties was found based in vitro measurements and Monte Carlo simulation of photon transport in tissue (Shepherd and Öberg et al 1990, Jakobsson and Nilsson 1993). LDF is based on the induced Doppler shift of light scattered from moving blood cells. The relative number of Doppler shifted photons, and their mean Doppler shift, are directly related to the concentration and root mean square velocity of red blood cells in the exposed area of tissue (Bonner and Nossal 1981).

The measurements were performed using a commercial laser Doppler perfusion monitor (PF5000, Perimed AB, Sweden) with two channels. The instrument has a semiconductor laser diode of $780 \mathrm{~nm}$ and a Doppler bandwidth of $20 \mathrm{~Hz}-13 \mathrm{kHz}$. Two probes were used in the experiment: (i) a small straight probe (Probe 407, Perimed AB) was used to measure the 
perfusion of the cooled location and (ii) a standard probe (Probe 408, Perimed AB) measured perfusion at the reference site. Each probe has illumination and detection fibers $250 \mu \mathrm{m}$ apart, with a core diameter of $125 \mu \mathrm{m}$ and a numerical aperture of 0.37 .

The laser Doppler instrument was allowed to warm up for 20 min before the start of the base measurements. The device in combination with the two probes was calibrated using a motility standard (PF 1000, Perimed AB). Both probes were attached to the measurement site with a probe holder and sticker. Perfusion measurements were taken at a sampling rate of $32 \mathrm{~Hz}$. The time constant of the output signals was set at $0.2 \mathrm{~s}$, which means low-pass filtering with a $3 \mathrm{~dB}$ cutoff frequency of $1.25 \mathrm{~Hz}$.

\subsection{Temperature measurements}

Four calibrated $\mathrm{J}$ type thermocouples were used in the experiments. One thermocouple was fixed with medical quality glue next to the perfusion probe on the scalp; another thermocouple was fixed to the outside of the cold cap. One thermocouple measured the skin temperature at the reference site. The last thermocouple was used to measure ambient temperature. Thermocouple measurements were taken at a sampling rate of $0.2 \mathrm{~Hz}$.

Skin temperature measurements are performed in a high temperature gradient setting. From previous theoretical work, we know that this gradient between the scalp skin and the cold cap can be as high as $1000{ }^{\circ} \mathrm{C} \mathrm{m}^{-1}$ (Janssen et al 2005). To ensure that the registered temperature is a good measure for the skin temperature, each thermocouple end was modified with a specially developed aluminum disk of radius $2 \mathrm{~mm}$ and thickness $0.5 \mathrm{~mm}$. This made the attachment to the scalp skin easier and, in combination with the medical quality glue, ensured that the thermal resistance between the thermocouple and the scalp skin was lower than the thermal resistance between the thermocouple and the cold cap.

\subsection{Experimental protocol}

All measurements were conducted with the subject sitting upright in an environment with an ambient temperature of approximately $20^{\circ} \mathrm{C}$. The subject was allowed to rest for $30 \mathrm{~min}$ before measurements to obtain a stable perfusion level. The scalp of the subject was cooled using a commercial scalp cooling system (Paxman Coolers Ltd, UK). It consists of a reservoir of coolant connected to a cap made from silicon tubing. At the beginning of the experiments, this reservoir was at room temperature. During 2 min before the start of a cooling experiment, temperature and perfusion of the scalp skin were measured to establish basal temperature and perfusion. These basal values were used to define the temperature difference and relative perfusion, such that measurements could be compared to each other.

The cooling experiment consisted of three stages. In the first stage the pump of the scalp cooling system is switched on without cooling the reservoir. This induced both pressure changes and small temperature changes in the cap. This stage lasted 5 min, and allowed us to discriminate between changes due to the difference in pressure exerted by the cap, and changes due to temperature. In the second stage, the cooling of the device was switched on for $90 \mathrm{~min}$. This resulted in a rate of scalp cooling that was slower than in clinical use (where the cap and reservoir are pre-cooled). In the final phase, the cooler of the system was switched off to enable the scalp to re-warm. Once again, the pump of the system remained pumping to avoid pressure changes on the skin. With this phase, it was possible to see if there are hysteresis effects in the relation between temperature and perfusion. The re-warming phase lasted $60 \mathrm{~min}$. Skin temperature and perfusion were continuously monitored during the experiment. 


\subsection{Biological zero measurements}

There is a residual signal in laser Doppler perfusion measurements from the tissue called biological zero (BZ), which is present even when there is no flow (Caspary et al 1988). It is suggested that this BZ signal should be subtracted from the normal perfusion value to get a better understanding of the perfusion, especially in the case of very low perfusion measurements (Fagrell 1990, Nilsson 1990, Kernick et al 1999). In addition, this BZ signal depends on the temperature of the tissue regarded (Kernick et al 1999). A previous study showed that during scalp cooling, perfusion drops to a stable low value that was higher than zero (Bülow et al 1985). Therefore, we expect that the BZ problem is relevant in our experiment. In general the BZ signal can be measured in vivo by an arterial occlusion with an inflated cuff. Unfortunately it is not possible to measure the BZ on the scalp because an occlusion is not possible. There is no other method for determining BZ on locations which are not accessible for occlusion. Hence the best possible way to find the influence of BZ on the minimum perfusion on scalp is to find $\mathrm{BZ}$ on a location of the body which can be occluded and use that data as the BZ of scalp skin.

We measured the BZ at a position on the forehand or palm which showed an LDF rest flux level comparable to that in the scalp. It was reported earlier that there is a difference in the BZ signal on different body locations with different rest flux (Abbot and Swanson-Beck 1993), but we expect that with the same rest flux level there would not be a significant difference in BZ between the scalp skin and the forehand. It is also important to investigate the influence of temperature on the $\mathrm{BZ}$ signal. We measured the $\mathrm{BZ}$ at normal body temperature and at the lowest temperature we reached during scalp cooling $\left(15^{\circ} \mathrm{C}\right)$ on nine healthy subjects (partly the same subjects participated for scalp perfusion measurements). The LDF probe was the same used in scalp perfusion measurements. The temperature was measured using a standard thermocouple. Normal perfusion (rest flux) and biological zero are measured at normal body temperature and at $15^{\circ} \mathrm{C}$. In order to cool down the skin at the measured spot to $15^{\circ} \mathrm{C}$, a water bath with ice was used in which the hand was immersed.

\subsection{Data analysis}

A moving average filter with a period of $5 \mathrm{~s}$ was used to filter out artifacts in the perfusion signal. This time period was chosen to match the sampling period of the thermocouple measurements. Using the base temperature $\left(T_{0}\right)$ and base perfusion $\left(W_{0}\right)$ determined in the first 5 min of each experiment, temperature difference $(\Delta T)$ and relative perfusion $(\varphi)$ were determined:

$$
\begin{aligned}
& \Delta T=T_{0}-T \\
& \varphi=W / W_{0} .
\end{aligned}
$$

The reductions in metabolism and subsequent reductions in perfusion due to falling temperatures are modeled according to a well-known $Q_{10}$ relation of thermal physiology. Due to a reduction in tissue temperature, there is a corresponding reduction in cell metabolism (Dennis et al 2003), and as a consequence the demand for nutrients (e.g. oxygen) will diminish. Subsequently it is no longer necessary to sustain current level of blood flow and the body reacts by lowering tissue perfusion (Fiala et al 1999). The reduction in metabolism and subsequent reduction in blood flow is by a factor $Q_{10}$ for every $10^{\circ} \mathrm{C}$ decrease in temperature. Relative perfusion may thus be written as

$$
\varphi=Q_{10}^{\left(-\Delta T / 10^{\circ} \mathrm{C}\right)},
$$

with factor $Q_{10}=2$ (Fiala et al 1999) up to 3 (Dennis et al 2003). 

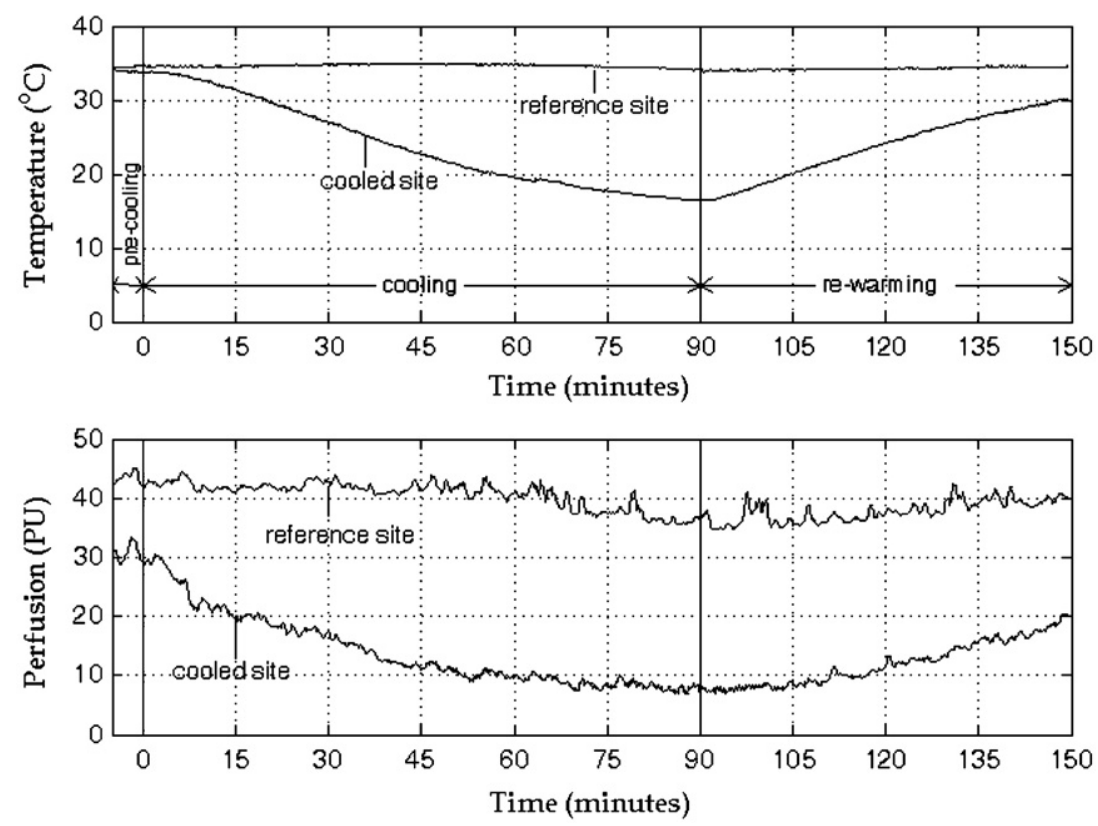

Figure 2. Typical time trace for temperature (top) and perfusion (bottom) of both the reference site and the cooling site.

It has alternatively been found that perfusion during scalp cooling decreases to a minimum value of about 20\% (Bülow et al 1985). Therefore, we investigated the goodness of fit of a minimum perfusion equation:

$$
\varphi=\varphi_{\min }+\left(1-\varphi_{\min }\right) \exp (-\Delta T / \Theta)
$$

where $\varphi_{\min }$ is the minimum relative perfusion value, and $\Theta$ is a constant that determines the rate at which perfusion drops.

Fits to the $Q_{10}$ equation (2) and minimum perfusion equation (3) were established using a nonlinear least-squares fitting routine (nlinfit.m) generally available in MATLAB (version 6.5.0, Release 13, The Mathworks, Inc.).

\section{Results}

\subsection{Relation between scalp skin temperature and perfusion}

A typical time trace for temperature and perfusion is shown in figure 2. Skin temperature drops from $34{ }^{\circ} \mathrm{C}$ to $16{ }^{\circ} \mathrm{C}$ in $90 \mathrm{~min}$ (cooling rate: $0.2^{\circ} \mathrm{C} \mathrm{min}{ }^{-1}$ ). Cutaneous blood flow drops from $30 \mathrm{PU}$ (perfusion units) to $8 \mathrm{PU}$. After the cooling stage, temperature returns to $30^{\circ} \mathrm{C}$ and perfusion to $20 \mathrm{PU}$. There are no significant reductions in either temperature or perfusion at the reference site.

Figure 3 shows the average relative perfusion $(\varphi)$ at the two positions (three experiments each) versus the temperature difference $(\Delta T)$. Inter-experiment variability (three experiments) is acceptable with an average standard deviation over the complete cooling range of 0.08 [-]. Scalp temperature and perfusion were reduced more at position $2\left(\Delta T_{\max }=22{ }^{\circ} \mathrm{C} ; \varphi_{\min }=\right.$ $0.21)$ than at position $1\left(\Delta T_{\max }=12{ }^{\circ} \mathrm{C} ; \varphi_{\min }=0.29\right)$. A two-tailed $t$-test was performed to 


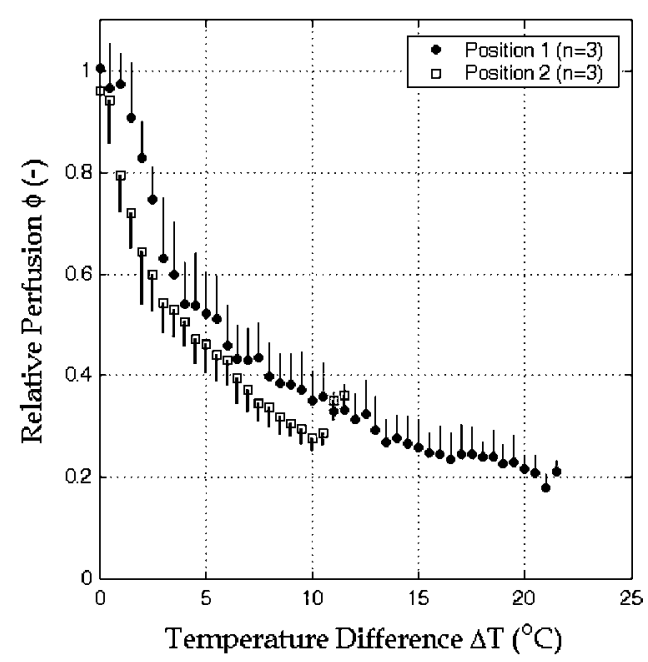

Figure 3. Average temperature difference and relative perfusion of position 1 (front of the head) and position 2 (top-right of the head) for one subject. Values represent averaged values of three combined experiments. Error bars denote standard deviation. $*$ : Position 1 significantly different from position $2(P<0.05)$.

check whether significant differences exist between the two positions. Except for two cooling temperatures $\left(\Delta T=1.0^{\circ} \mathrm{C}\right.$ and $\left.\Delta T=1.5^{\circ} \mathrm{C}\right)$ relative perfusion did not differ significantly between the two positions $(p<0.05)$. The results of these experiments indicate that both inter-position and inter-experiment variability are low.

The achieved skin temperature drop for the nine subjects ranged from $7{ }^{\circ} \mathrm{C}$ to $22{ }^{\circ} \mathrm{C}$ (figure 4). Lowest relative perfusion for the nine subjects ranged from $21 \%$ to $44 \%$. According to a two-tailed $t$-test, there are no hysteresis effects that come into play during the transition between the cooling and the re-warming stage $(p<0.05)$.

We used the data of each individual response to fit the parameters of equations (2) and (3) using a nonlinear fitting routine. This resulted in $Q_{10}=3.5 \pm 1.5$ (mean \pm standard deviation) for equation (2) and $\varphi_{\min }=0.27 \pm 0.08$ for equation (3). The range in parameters was $2.1<$ $Q_{10}<5.9$ for equation (2) and $0.16<\varphi_{\min }<0.41$.

An averaged response of the nine individuals has been calculated (figure 5) to establish a relationship between the temperature difference and relative perfusion. In this calculation, both the cooling and the re-warming stages were used. Relative perfusion starts at a value of $89 \%$ at $\Delta T=0.25^{\circ} \mathrm{C}$ and with further cooling $\left(\Delta T=21^{\circ} \mathrm{C}\right)$ a minimum perfusion value of $29 \%$ is reached.

Accumulated data of all nine subjects were used to fit the parameters of equations (2) and (3) using a nonlinear fitting routine. This resulted in $Q_{10}=2.5\left(R^{2}=0.68\right)$ with a 95\% confidence interval $\left(\mathrm{CI}_{95}\right)$ of 2.33-2.79 for equation (2) and $\varphi_{\min }=0.29\left(0.28<\mathrm{CI}_{95}<\right.$ $0.30)$ and $\Theta=4{ }^{\circ} \mathrm{C}\left(3.85<\mathrm{CI}_{95}<4.38\right)\left(R^{2}=0.8\right)$ for equation (3) (figure 5). The fitted $Q_{10}$ value is in the range of values cited in the literature. A good fit is impossible due to the observed behavior in our experiments that perfusion levels off at a value of about $25 \%$ instead of continuing toward zero. Hence the fit function underestimates relative perfusion for strong cooling $\left(\Delta T>12{ }^{\circ} \mathrm{C}\right)$ while overestimating perfusion for low and mild cooling. The minimum perfusion function gives a much better fit to the data over the entire cooling range. The value of rate constant $\Theta=4{ }^{\circ} \mathrm{C}$ signifies that $95 \%$ of the drop in perfusion is reached when the temperature difference equals $\Delta T=12{ }^{\circ} \mathrm{C}$. 

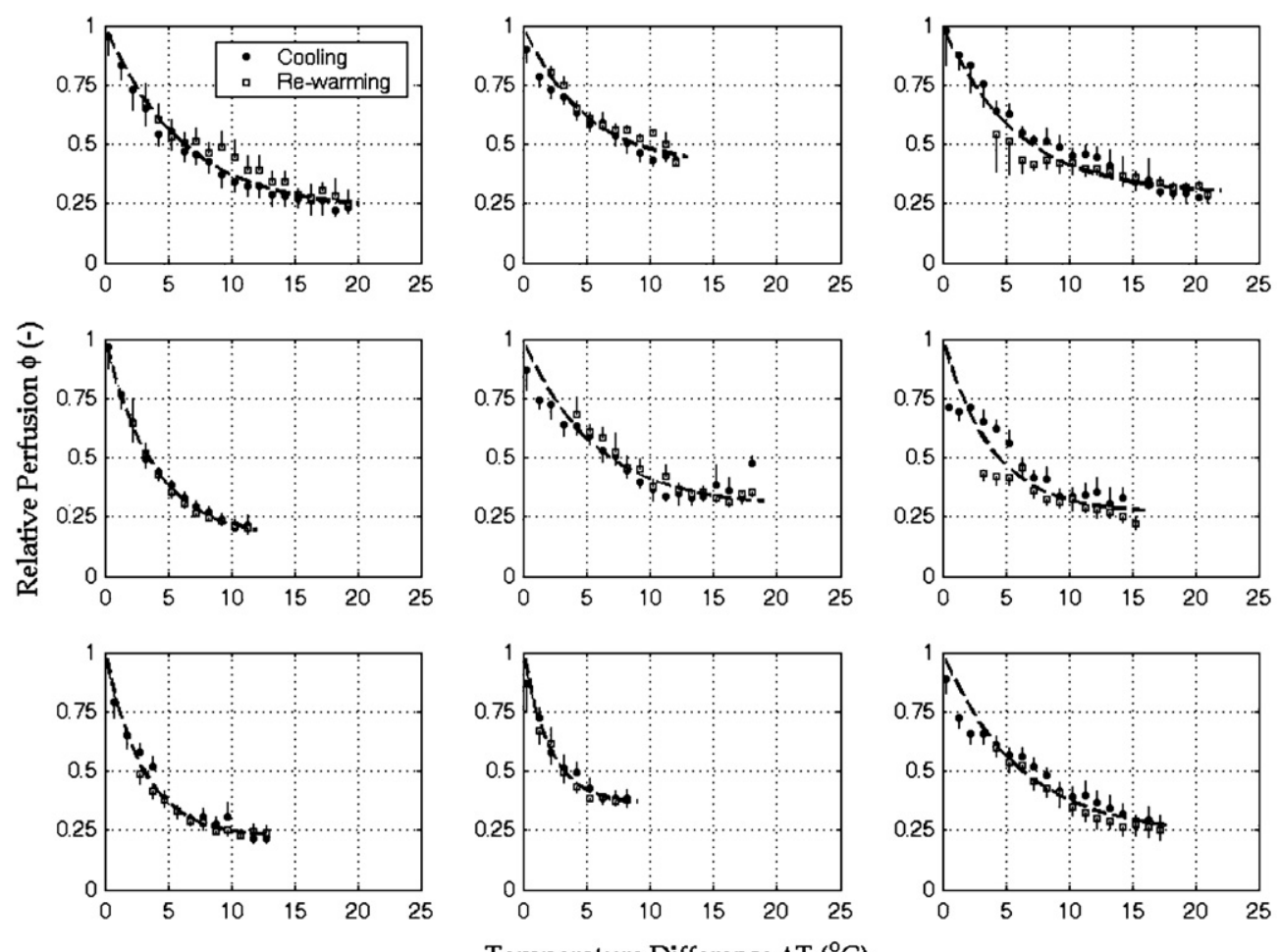

Temperature Difference $\Delta \mathrm{T}\left({ }^{\circ} \mathrm{C}\right)$

Figure 4. Average temperature difference and relative perfusion of each individual subject for both the cooling $(\bullet)$ and re-warming $(\square)$ stage. Error bars represent standard deviation. The dashed line represents the best fit for the minimum perfusion function.

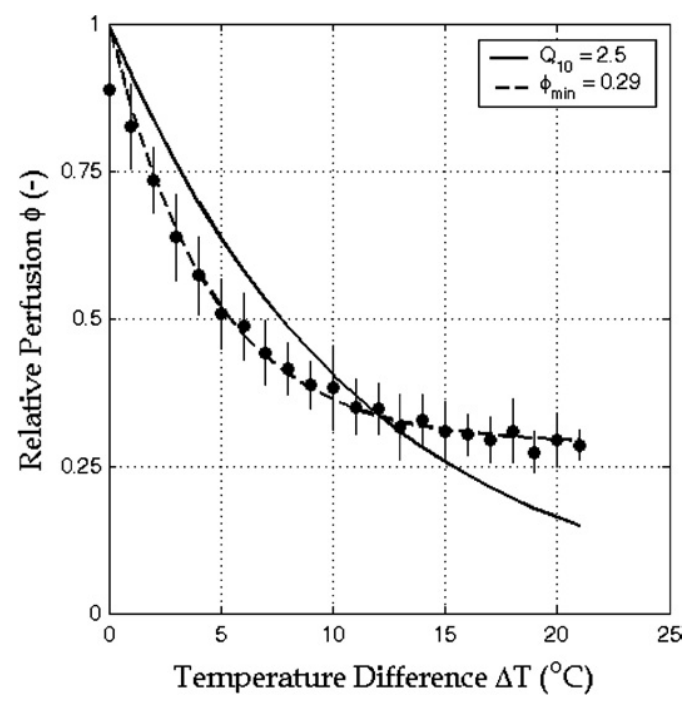

Figure 5. Average temperature difference and relative perfusion and best fits for $Q_{10}$ and minimum perfusion $\left(\theta_{\mathrm{min}}\right)$. Error bars represent standard error. 


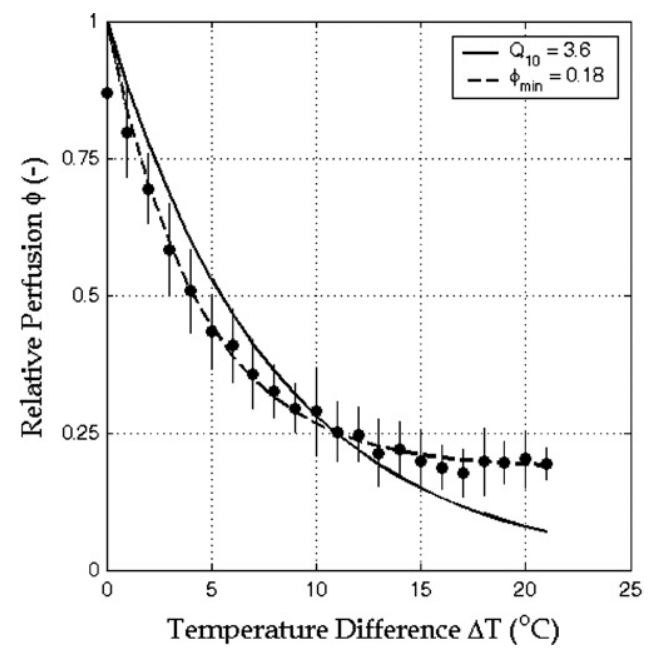

Figure 6. Average relative temperature difference and relative perfusion and best fits for $Q_{10}$ and minimum perfusion $\left(\theta_{\mathrm{min}}\right)$ corrected for biological zero. Error bars represent standard error.

\subsection{Influence of biological zero (BZ)}

The rest flux at body temperature for nine subjects in perfusion units (PU) - with the range given in brackets-was 29.1 PU (24.7-34.4) and the mean BZ was 5.29 PU (2.65-7.42). At $15^{\circ} \mathrm{C}$ the mean perfusion was $10.5 \mathrm{PU}$ (7.06-14.79) and the mean BZ was 4.50 PU (2.585.39). In comparing the PU obtained from different persons one should be aware of the fact that perfusion can vary intra- and interindividually. The variation of optical properties will affect the mean sampling depth and can influence the rest flux (Larsson et al 2001). Ideally speaking, individual BZ levels measured on the same location should be used in the analysis of perfusion data. However, since in this case no local BZ levels could be measured, a BZ correction has to be based on an average value. Since we choose measurement locations having comparable rest flux in all individuals we expect that the influence of optical properties on our measurements is not significant.

The results show that the difference in BZ between normal skin temperature and $15^{\circ} \mathrm{C}$ is not significant. This confirms the earlier report on the BZ variation in the temperature range we used (Abbot and Swanson-Beck 1993). The measured mean biological zero (4.5 PU) was significantly less than the average minimum perfusion value (11.2 PU) we found in our measurements. We investigated the influence of BZ on our results by subtracting the BZ and fitting our model function from the average perfusion in figure 5. For this we used the average $\mathrm{BZ}$ measured on the hand at the minimum temperature we acquired during scalp cooling, $\mathrm{BZ}=4.5$. With this correction for the BZ, a fit of the parameters in equations (2) and (3) to the accumulated data of all nine subjects resulted in $Q_{10}=3.6$ and $\varphi_{\min }=0.18$ (figure 6). This shows that the minimum perfusion value still exists even after correcting for BZ.

\section{Discussion and conclusion}

We have investigated the relationship between temperature and perfusion on the human scalp skin with thermocouples and laser Doppler perfusion probes. The single-subject repetitive experiments showed that the measurements are reproducible, and that no differences exist between the two different positions regarded. We were not able to cool the position on the 
right-hand side as much as the central position, which can be attributed to better contact between the skin and the cap at the central position for this head/cap combination. Computations have shown a high sensitivity of skin temperature to the gap thickness between the skin and the cap (Janssen et al 2005). The experiments on the nine subjects showed that cooling the scalp reduced perfusion down to $20-40 \%$. Re-warming resulted in an increase in perfusion. In a previous study (Bülow et al 1985), blood flow remained significantly lower than the pre-cooling level during the re-warming phase. However, in our study there were no significant differences between the cooling phase and the re-warming phase, which suggests that hysteresis effects of this kind do not play a role in this experiment. We found that we were not able to cool each person to the same degree. This is probably caused by anatomical differences such as head shape and thickness of the insulating fat layer, two factors that were found to have a high influence on scalp skin temperature during scalp cooling (Janssen et al 2005). In contrast, the same study showed that physiological variations in scalp skin perfusion were far less influential.

The data were used to establish a relationship between scalp skin temperature and cutaneous perfusion. Perfusion does not exponentially go to zero, instead an exponential decrease with $\Theta=4{ }^{\circ} \mathrm{C}$ to a relative perfusion level of 0.29 described the data well.

Since the minimum perfusion we measured at lower temperatures is significantly higher than the measured biological zero at this temperature at another location with a comparable rest flux value, the biological zero variations would not affect our findings of a relation between temperature and scalp skin perfusion.

The minimum perfusion we cite in our measurements (also after correcting for BZ) shows that cooling beyond $\Delta T \approx 3 \Theta=12{ }^{\circ} \mathrm{C}$ does not significantly reduce the supply of the drug to the skin any further. The latter finding is a surprising outcome suggesting that below a certain critical temperature, lower skin temperatures do not significantly reduce the supply of the drug to the skin any further. The consequence of this is that lowering skin temperature beneath this critical temperature might only be useful for reducing local tissue metabolism. This is something that has to be taken into account in the protocol for scalp cooling during administration of chemotherapy. Currently, we are investigating the effect of temperature on doxorubicin uptake and metabolism using in vitro experiments.

\section{Acknowledgments}

The Eindhoven University of Technology was sponsored by SOBU, an administrative cooperation between Tilburg University and the Eindhoven University of Technology. The University of Twente was supported by the Netherlands Technology Foundation STW (grant 5840). The authors are grateful to Perimed AB and Paxman Ltd for providing instrumentation.

\section{References}

Abbot N C and Swanson-Beck J 1993 Biological zero in laser Doppler measurements in normal, ischaemic and inflamed human skin Int. J. Microcirc. Clin. Exp. 2 89-98

Bonner R F and Nossal R 1981 Model for laser Doppler measurements of blood flow in tissue Appl. Opt. 20 2097-107 Bülow J, Friberg L, Gaardsting O and Hansen M 1985 Frontal subcutaneous blood flow, and epi- and subcutaneous temperatures during scalp cooling in normal man Scand. J. Clin. Lab. Invest. 45 505-8

Cash T F 2001 The psychology of hair loss and its implications for patient care Clin. Dermatol. 19 161-6

Caspary L, Creutzig A and Alexander K 1988 Biological zero in laser Doppler fluximetry Int. J. Microcirc. Clin. Exp. $7367-71$

Dennis B H, Eberhart R C, Dulikravich G S and Radons S W 2003 Finite-element simulation of cooling of realistic 3D human head and neck J. Biomech. Eng. $125832-40$ 
Fagrell B 1990 Perimed's LDV flowmeter Laser-Doppler Blood Flowmetry ed A P Shepherd and P Å Öberg (Boston: Kluwer Academic)

Fiala D, Lomas K J and Stohre M 1999 A computer model of human thermoregulation for a wide range of environmental conditions: the passive system J. Appl. Phys. 87 1957-72

Friedman E A, Harris P A, Wood A J, Stein C M and Kurnik D 2007 The effects of tadalafil on cold-induced vasoconstriction in patients with Raynaud's phenomenon Clin. Pharmacol. Ther. 81 503-9

Grevelman E G and Breed W P M 2005 Prevention of chemotherapy-induced hair loss by scalp cooling Ann. Oncol. $16352-8$

Jakobsson A and Nilsson G 1993 Prediction of sampling depth and photon pathlength in laser Doppler flowmetry Med. Biol. Eng. Comput. 31 301-7

Janssen F E M, Van Leeuwen G M J and Van Steenhoven A A 2005 Modelling of temperature and perfusion during scalp cooling Phys. Med. Biol. 50 4065-73

Kanetaka T, Komiyama T, Onozuka A, Miyata T and Shigematsu H 2004 Laser Doppler skin perfusion pressure in the assessment of Raynaud's phenomenon Eur. J. Vasc. Endovasc. Surg. 27 414-6

Kernick D P, Tooke J E and Shore A C 1999 The biological zero signal in laser doppler fluximetry-origins and practical implications Pflügers Arch.—Eur. J. Physiol. 437 624-31

Larsson M, Steenbergen W and Strömberg T 2001 Influence of optical properties and fibre separation on laser Doppler flowmetry J. Biomed. Opt. 7 236-43

Lau C S, Khan F, Brown R, McCallum P and Belch J J 1995 Digital blood flow response to body warming, cooling, and rewarming in patients with Raynaud's phenomenon Angiology 46 1-10

Maniewski R, Leger P, Lewandowski P, Liebert A, Bendayan P, Boccalon H, Bajorski L and Moller K O 1999 Spectral analysis of laser-Doppler perfusion signal measured during thermal test Technol. Health Care 7 163-9

Nilsson G E 1990 Peripheral vascular diseases Laser-Doppler Blood Flowmetry ed A P Shepherd and P $\AA$ Öberg (Boston: Kluwer Academic)

Pèrgola P E, Kellog D L, Johnson J M, Kosiba W A and Solomon D E 1993 Role of sympathetic nerves in the vascular effects of local temperature in human forearm skin Am. J. Phys. 265 H785-92

Popivanov D, Mineva A, Bendayan P, Leger P, Boccalon H and Moller K O 1999 Dynamic characteristics of laserDoppler flux in normal individuals and patients with Raynaud's phenomenon before and after treatment with nifedipine under different thermal conditions Technol. Health Care 7 193-203

Protière C, Evans K, Camerlo J, d'Ingrado M P, Macquart-Moulin G, Viens P, Maraninchi D and Genre D 2002 Efficacy and tolerance of a scalp-cooling system for prevention of hair loss and the experience of breast cancer patients treated by adjuvant chemotherapy Support. Care Cancer 10 529-37

Shepherd A P and Öberg P Å (ed) 1990 Laser-Doppler Blood Flowmetry (Boston: Kluwer Academic)

Thompson C S, Holowatz L A and Kenney W L 2005 Attenuated noradrenergic sensitivity during local cooling in aged human skin J. Physiol. (Lond.) 564 313-9 